Annales Academiæ Scientiarum Fennicæ

Mathematica

Volumen 38, 2013, 341-350

\title{
EXTRAPOLATION PROPERTIES OF CLOSED OPERATOR IDEALS
}

\author{
Luz M. Fernández-Cabrera and Antón Martínez \\ Universidad Complutense de Madrid, Departamento de Matemática Aplicada \\ 28040 Madrid, Spain; luz_fernandez-c@mat.ucm.es \\ Universidad de Vigo, Departamento de Matemática Aplicada I \\ 36200 Vigo, Spain; antonmar@uvigo.es
}

\begin{abstract}
We investigate the extrapolation properties of operator ideals $\mathcal{A}$ whose components $\mathcal{A}(E, F)$ are closed subspaces of $\mathcal{L}(E, F)$. In particular, results apply to weakly compact operators, Banach-Saks operators, Rosenthal operators and Asplund operators.
\end{abstract}

\section{Introduction}

Interpolation theory and extrapolation theory are complementary subjects, with applications in the study of boundedness of important operators and to the research on function spaces. See, for example, the books by Stein [26], Bergh and Löfström [3], Triebel [28, 29], Milman [21] and Edmunds and Triebel [12]. Interpolation theory has also found deep applications in operator theory, as can be seen in the monographs by Triebel [28] and Pietsch [25], or in the paper by Heinrich [16]. But the connection of extrapolation theory with operator theory has not yet been fully studied.

As far as we know, Triebel [30] used by the first time extrapolation ideas to study the degree of compactness of certain limiting Sobolev embeddings. Abstract results in this direction have been established by Kühn and Schonbek [19] and Cobos and Kühn [9]. On the other hand, Kryczka [20] and Nikolova and Zachariades [24] have investigated the extrapolation properties of weakly compact operators. The case of compact operators has been considered by Cobos and Kühn [8].

Weakly compact operators and compact operators are example of closed operator ideals. So, it is natural to study if there are general results which are valid for this kind of operator ideals. This is the aim of the paper. Our results apply to the two operator ideals mentioned before but also to several others, among them BanachSaks operators, Rosenthal operators and Asplund operators, also referred to as dual Radon-Nikodym operators or decomposing operators. Moreover, for the case of weakly compact operators, we can improve some results of [24].

The paper is organized as follows. In Section 2 we review some general facts on closed operator ideals, as well as the basic constructions of extrapolation theory. In Section 3, we prove results for the special case when we only extrapolate either the source spaces of the operator, or the target spaces. Finally, in Section 4, we consider the general case when extrapolation takes place both in the source spaces and in the target spaces. For these last results, we require that the ideal satisfies the so-called $\Sigma_{q}$-condition. Hence, they do not apply to compact operators.

doi:10.5186/aasfm.2013.3821

2010 Mathematics Subject Classification: Primary 47L20, 46B70, 47B10.

Key words: Closed operator ideals, weakly compact operators, extrapolation of operators, logarithmic spaces.

The authors have been supported in part by the Spanish Ministerio de Economía y Competitividad (MTM2010-15814). 


\section{Preliminaries}

Subsequently, letters $X, Y, E, F$ stand for Banach spaces. As usual, we write $U_{X}$ for the closed unit ball of $X$ and $\mathcal{L}(X, Y)$ for the space of all bounded linear operators from $X$ into $Y$, endowed with the operator norm. An operator ideal $\mathcal{A}$ is a class of bounded linear operators such that each component $\mathcal{A} \cap \mathcal{L}(X, Y)=\mathcal{A}(X, Y)$ is a linear subspace of $\mathcal{L}(X, Y)$ which contains the finite rank operators, and it satisfies that $S T R \in \mathcal{A}(X, Y)$ whenever $R \in \mathcal{L}(X, E), T \in \mathcal{A}(E, F)$ and $S \in \mathcal{L}(F, Y)$.

The operator ideal $\mathcal{A}$ is said to be closed if the components $\mathcal{A}(X, Y)$ are closed subspaces of $\mathcal{L}(X, Y)$. Many classical ideals are closed. For example, compact operators $\mathcal{K}$ and weakly compact operators $\mathcal{W}$ have this property.

An ideal $\mathcal{A}$ is called injective if for every isomorphic embedding $j \in \mathcal{L}(Y, F)$ and every $T \in \mathcal{L}(X, Y)$ it follows from $j T \in \mathcal{A}(X, F)$ that $T \in \mathcal{A}(X, Y)$. The ideal $\mathcal{A}$ is said to be surjective if for every surjection $Q \in \mathcal{L}(E, X)$ and every $T \in \mathcal{L}(X, Y)$ it follows from $T Q \in \mathcal{A}(E, Y)$ that $T \in \mathcal{A}(X, Y)$. Both ideals $\mathcal{K}$ and $\mathcal{W}$ are injective and surjective. Strictly singular operators form an ideal which is closed and injective but it is not surjective. On the other hand, the ideal of strictly cosingular operators is closed and surjective but it is not injective. We refer to [25] and [11] for more details.

Let $\mathcal{A}$ be an injective closed operator ideal. The following characterization holds (see [18], [31]): An operator $T \in \mathcal{L}(X, Y)$ belongs to $\mathcal{A}$ if and only if given any $\delta>0$, there is a Banach space $F$ and an operator $R \in \mathcal{A}(X, F)$ such that

$$
\|T x\|_{Y} \leq \delta\|x\|_{X}+\|R x\|_{F}, \quad x \in X .
$$

For a surjective closed operator ideal $\mathcal{A}$, it turns out that the necessary and sufficient condition for $T \in \mathcal{L}(X, Y)$ to belong to $\mathcal{A}$ is that for any $\delta>0$, there is a Banach space $E$ and an operator $S \in \mathcal{A}(E, Y)$ such that

$$
T\left(U_{X}\right) \subseteq \delta U_{Y}+S\left(U_{E}\right),
$$

(see [1]). More details on these characterizations can be found in [7] and [14], where the ideal measures that they define are studied and their interpolation properties are shown (see also [27] for the case of the ideal $\mathcal{W}$ ).

In what follows, let $\Theta$ be an interval $\Theta \subseteq[0,1]$, let $Y_{0}, Y_{1}$ be Banach spaces and let $Y_{\eta}, \eta \in \Theta$, be an ordered family of Banach spaces, that is to say

$$
Y_{0} \hookrightarrow Y_{\theta} \hookrightarrow Y_{\eta} \hookrightarrow Y_{1} \text { for any } \theta \leq \eta \text { with } \theta, \eta \in \Theta .
$$

Here $\hookrightarrow$ means continuous embedding. We suppose that embedding operators are uniformly bounded.

The following extrapolation spaces are considered in $[17,13,15,6,5]$ and [8] among other papers. Subsequently, given positive functions $f, g$ defined on an interval $I$, we write $f(t) \sim g(t)$ if there are constants $c_{1}, c_{2}>0$ such that $c_{1} f(t) \leq g(t) \leq$ $c_{2} f(t)$ for all $t \in I$.

Definition 2.1. Let $1 \leq q \leq \infty$ and let $0 \leq \theta<1$ such that for some $\varepsilon>0$, we have $(\theta, \theta+\varepsilon) \subseteq \Theta$. Let $\varphi$ be a positive, continuous and monotone function on $(0, \varepsilon)$ such that $\varphi(t) \sim \varphi(2 t)$ and

$$
\left(\int_{0}^{\varepsilon} \varphi(t)^{q} \frac{d t}{t}\right)^{1 / q}<\infty
$$


(where, as usual, the integral should be replaced by the supremum if $q=\infty$ ). The space $Y_{\theta}(\log Y)_{\varphi, q}^{+}=Y_{\theta, \varphi, q}^{+}$consists of all those $y \in \bigcap_{\theta<\eta<\theta+\varepsilon} Y_{\eta}$ which have a finite norm

$$
\|y\|_{Y_{\theta}(\log Y)_{\varphi, q}^{+}}=\left(\int_{0}^{\varepsilon}\left(\varphi(t)\|y\|_{Y_{\theta+t}}\right)^{q} \frac{d t}{t}\right)^{1 / q} .
$$

Let $J \in \mathbf{N}$ with $2^{-J}<\varepsilon$ and write

$$
\sigma_{k}=\theta+2^{-k}, \quad k \geq J .
$$

Then $\|\cdot\|_{Y_{\theta}(\log Y)_{\varphi, q}^{+}}$is equivalent to

$$
\|y\|_{Y_{\theta, \varphi, q}^{+}}=\left(\sum_{n=J}^{\infty}\left(\varphi\left(2^{-n}\right)\|y\|_{Y_{\sigma_{n}}}\right)^{q}\right)^{1 / q}
$$

(see [8]). We also notice that

$$
Y_{\theta}(\log Y)_{\varphi, q}^{+} \hookrightarrow Y_{\eta} \text { for any } \theta<\eta<\theta+\varepsilon,
$$

and that spaces $Y_{\theta}(\log Y)_{\varphi, q}^{+}$do not depend on the choice of $\varepsilon>0$. Furthermore, a change in the value of $J \in \mathbf{N}$ with $2^{-J}<\varepsilon$ yields an equivalent norm to $\|\cdot\|_{Y_{\theta}(\log Y)_{\varphi, q}^{+}}$.

Definition 2.2. Let $1 \leq q \leq \infty, 1 / q+1 / q^{\prime}=1$ and let $0<\theta \leq 1$ such that for some $\varepsilon>0$, we have $(\theta-\varepsilon, \theta) \subseteq \Theta$. Let $\psi$ be a positive, continuous and monotone function on $(0, \varepsilon)$ such that $\psi(t) \sim \psi(2 t)$ and

$$
\left(\int_{0}^{\varepsilon} \psi(t)^{-q^{\prime}} \frac{d t}{t}\right)^{1 / q^{\prime}}<\infty
$$

The space $Y_{\theta}(\log Y)_{\psi, q}^{-}=Y_{\theta, \psi, q}^{-}$is formed by all those $y \in Y_{1}$ which can be represented as $y=\int_{0}^{\varepsilon} w(t) d t / t$ (convergence in $Y_{1}$ ) with $w(t) \in Y_{\theta-t}$ and

$$
\left(\int_{0}^{\varepsilon}\left(\psi(t)\|w(t)\|_{Y_{\theta-t}}\right)^{q} \frac{d t}{t}\right)^{1 / q}<\infty .
$$

We provide $Y_{\theta}(\log Y)_{\bar{\psi}, q}^{-}$with the norm $\|\cdot\|_{Y_{\theta}(\log Y)_{\bar{\psi}, q}^{-}}$defined by the infimum of the values (2.7) over all possible representations of $y$ of the above type.

Let $J \in \mathbf{N}$ with $2^{-J}<\varepsilon$. Write

$$
\lambda_{k}=\theta-2^{-k}, \quad k \geq J .
$$

Then $y \in Y_{\theta}(\log Y)_{\psi, q}^{-}$if and only if there exist $y_{n} \in Y_{\lambda_{n}}, n \geq J$, such that $y=$ $\sum_{n=J}^{\infty} y_{n}$ (convergence in $Y_{1}$ ) and

$$
\left(\sum_{n=J}^{\infty}\left(\psi\left(2^{-n}\right)\left\|y_{n}\right\|_{Y_{\lambda_{n}}}\right)^{q}\right)^{1 / q}<\infty
$$

Moreover,

$$
\|y\|_{Y_{\theta, \psi, q}^{-}}=\inf \left\{\left(\sum_{n=J}^{\infty}\left(\psi\left(2^{-n}\right)\left\|y_{n}\right\|_{Y_{\lambda_{n}}}\right)^{q}\right)^{1 / q}: y=\sum_{n=J}^{\infty} y_{n}\right\}
$$

is an equivalent norm to $\|\cdot\|_{Y_{\theta}(\log Y)_{\psi, q}^{-}}$(see [8]). 
Again $Y_{\theta}(\log Y)_{\psi, q}^{-}$does not depend on the choice of $\varepsilon>0$ and this time we have

$$
Y_{\eta} \hookrightarrow Y_{\theta}(\log Y)_{\psi, q}^{-} \text {for any } \theta-\varepsilon<\eta<\theta .
$$

For $b>0$, if $\varphi(t)=t^{b}$ and $\psi(t)=t^{-b}$, we denote the extrapolation spaces by $Y_{\theta}(\log Y)_{b, q}^{+}=Y_{\theta, b, q}^{+}$and $Y_{\theta}(\log Y)_{b, q}^{-}=Y_{\theta, b, q}^{-}$, respectively.

\section{Extrapolation either on the source spaces or on the domain spaces}

We start with a result regarding extrapolation on the source spaces of the operator.

Theorem 3.1. Let $\mathcal{A}$ be an injective closed operator ideal, let $X$ be a Banach space and let $\left\{Y_{\eta}\right\}_{\eta \in \Theta}, \theta, q, \varepsilon$ and $\varphi$ as in Definition 2.1. Let $J \in \mathbf{N}$ such that $2^{-J}<\varepsilon$ and let $\sigma_{n}$ be the numbers defined in (2.4). Suppose that $T: X \rightarrow \bigcap_{\theta<\eta<\theta+\varepsilon} Y_{\eta}$ is a linear operator satisfying that

$$
\begin{cases}\left(\sum_{n=J}^{\infty}\left(\varphi\left(2^{-n}\right)\|T\|_{X, Y_{\sigma_{n}}}\right)^{q}\right)^{1 / q}<\infty & \text { if } 1 \leq q<\infty \\ \lim _{n \rightarrow \infty} \varphi\left(2^{-n}\right)\|T\|_{X, Y_{\sigma_{n}}}=0 & \text { if } \quad q=\infty\end{cases}
$$

If $T \in \mathcal{A}\left(X, Y_{\eta}\right)$ for any $\theta<\eta<\theta+\varepsilon$, then $T \in \mathcal{A}\left(X, Y_{\theta, \varphi, q}^{+}\right)$.

Proof. We shall work with the characterization of operators belonging to $\mathcal{A}$ given by (2.1) and with the discrete norm $\|\cdot\|_{Y_{\theta, \varphi, q}^{+}}$of $Y_{\theta, \varphi, q}^{+}$. Take any $\delta>0$. Using (3.1) we can find $N \in \mathbf{N}, N \geq J$ such that

$$
\left(\sum_{n=N+1}^{\infty}\left(\varphi\left(2^{-n}\right)\|T\|_{X, Y_{\sigma_{n}}}\right)^{q}\right)^{1 / q} \leq \frac{\delta}{2}
$$

Since $T \in \mathcal{A}\left(X, Y_{\sigma_{k}}\right)$ for $k=J, \ldots, N$, there are Banach spaces $F_{k}$ and operators $R_{k} \in \mathcal{A}\left(X, F_{k}\right)$ such that

$$
\|T x\|_{Y_{\sigma_{k}}} \leq \frac{\delta}{2 \varphi\left(2^{-k}\right) N^{1 / q}}\|x\|_{X}+\left\|R_{k} x\right\|_{F_{k}}, \quad x \in X .
$$

Let $F=\bigoplus_{k=J}^{N} F_{k}$, with the norm

$$
\left\|\left(z_{J}, \ldots, z_{N}\right)\right\|_{F}=\left(\sum_{k=J}^{N}\left(\varphi\left(2^{-k}\right)\left\|z_{k}\right\|_{F_{k}}\right)^{q}\right)^{1 / q},
$$

and let $R: X \rightarrow F$ be the operator defined by

$$
R x=\left(R_{J} x, \ldots, R_{N} x\right) .
$$

One can easily check that $R \in \mathcal{A}(X, F)$. For any $x \in X$, we obtain by (3.2) and (3.3) that

$$
\begin{aligned}
\|T x\|_{Y_{\theta, \varphi, q}^{+}} & \leq\left(\sum_{n=J}^{N}\left(\varphi\left(2^{-n}\right)\|T x\|_{Y_{\sigma_{n}}}\right)^{q}\right)^{1 / q}+\left(\sum_{n=N+1}^{\infty}\left(\varphi\left(2^{-n}\right)\|T x\|_{Y_{\sigma_{n}}}\right)^{q}\right)^{1 / q} \\
& \leq\left(\sum_{n=J}^{N}\left(\frac{\delta}{2}\right)^{q} \frac{1}{N}\right)^{1 / q}\|x\|_{X}+\left(\sum_{n=J}^{N}\left(\varphi\left(2^{-n}\right)\left\|R_{k} x\right\|_{F_{k}}\right)^{q}\right)^{1 / q}+\frac{\delta}{2}\|x\|_{X} \\
& \leq \delta\|x\|_{X}+\|R x\|_{F} .
\end{aligned}
$$


Hence, (2.1) implies that $T \in \mathcal{A}\left(X, Y_{\theta, \varphi, q}^{+}\right)$.

Next we consider the case where extrapolation takes place in domain spaces. Subsequently, given a Banach space $Y$ and $\lambda>0$, we designate by $\lambda Y$ the space $Y$ normed by $\|\cdot\|_{\lambda Y}=\lambda\|\cdot\|_{Y}$.

Theorem 3.2. Let $\mathcal{A}$ be a surjective closed operator ideal, let $X$ be a Banach space and let $\left\{Y_{\eta}\right\}_{\eta \in \Theta}, \theta, q, \varepsilon$ and $\psi$ as in Definition 2.2. Take $J \in \mathbf{N}$ with $2^{-J}<\varepsilon$ and let $\lambda_{n}$ be the numbers given by (2.8). Assume that $T: Y_{1} \rightarrow X$ is a bounded linear operator such that

$$
\begin{cases}\left(\sum_{n=J}^{\infty}\left(\|T\|_{Y_{\lambda_{n}, X}} / \psi\left(2^{-n}\right)\right)^{q^{\prime}}\right)^{1 / q^{\prime}}<\infty & \text { if } 1<q \leq \infty \\ \lim _{n \rightarrow \infty}\|T\|_{Y_{\lambda_{n}, X}} / \psi\left(2^{-n}\right)=0 & \text { if } \quad q=1 .\end{cases}
$$

If $T \in \mathcal{A}\left(Y_{\eta}, X\right)$ for any $\theta-\varepsilon<\eta<\theta$, then $T \in \mathcal{A}\left(Y_{\theta, \psi, q}^{-}, X\right)$.

Proof. The relevant characterization for operators of $\mathcal{A}$ is now (2.2). Take any $\delta>0$ and let $J \in \mathbf{N}$ with $2^{-J}<\varepsilon$. By (3.4), there exists $N \in \mathbf{N}, N \geq J$, such that

$$
\left(\sum_{n=N+1}^{\infty}\left(\|T\|_{Y_{\lambda_{n}}, X} / \psi\left(2^{-n}\right)\right)^{q^{\prime}}\right)^{1 / q^{\prime}} \leq \frac{\delta}{4}
$$

For $J \leq k \leq N$, take positive numbers $\delta_{k}$ with $\sum_{k=J}^{N} \delta_{k}=\delta / 2$. Since $T \in$ $\mathcal{A}\left(\frac{\psi\left(2^{-k}\right)}{2} Y_{\lambda_{k}}, X\right)$, there exist Banach spaces $E_{k}$ and operators $R_{k} \in \mathcal{A}\left(E_{k}, X\right)$ such that

$$
T\left(U_{\frac{\psi(2-k)}{2} Y_{\lambda_{k}}}\right) \subseteq \delta_{k} U_{X}+R_{k}\left(U_{E_{k}}\right), \quad J \leq k \leq N
$$

Put $E=\bigoplus_{k=J}^{N} E_{k}$, endowed with the norm

$$
\left\|\left(z_{J}, \ldots, z_{N}\right)\right\|_{E}=\sup _{J \leq k \leq N}\left\|z_{k}\right\|_{E_{k}}
$$

and let $S: E \rightarrow X$ be the operator defined by

$$
R\left(z_{J}, \ldots, z_{N}\right)=\sum_{k=J}^{N} R_{k}\left(z_{k}\right) .
$$

It is not hard to check that $R \in \mathcal{A}(E, X)$. Now take any $y \in U_{Y_{\theta, \psi}^{-},}$. We can find a representation $y=\sum_{n=J}^{\infty} y_{n}$ (convergence in $Y_{1}$ ) with $y_{n} \in Y_{\lambda_{n}}$ and

$$
\left(\sum_{n=J}^{\infty}\left(\psi\left(2^{-n}\right)\left\|y_{n}\right\|_{Y_{\lambda_{n}}}\right)^{q}\right)^{1 / q} \leq 2
$$

For $J \leq k \leq N$, since $\left\|y_{k}\right\|_{\left(\psi\left(2^{-k}\right) / 2\right) Y_{\lambda_{k}}} \leq 1$, by (3.6) there is $z_{k} \in U_{E_{k}}$ such that $\left\|T y_{k}-R_{k} z_{k}\right\|_{X} \leq \delta_{k}$. Put $z=\left(z_{J}, \ldots, z_{N}\right)$. Then $z \in U_{E}$. Using Hölder's inequality and (3.5), we derive

$$
\|T y-R z\|_{X} \leq\left\|\sum_{n=J}^{N} T y_{k}-\sum_{n=J}^{N} R_{k} z_{k}\right\|_{X}+\left\|\sum_{n=N+1}^{\infty} T y_{n}\right\|_{X}
$$




$$
\begin{aligned}
& \leq \sum_{n=J}^{N} \delta_{k}+\sum_{n=N+1}^{\infty}\|T\|_{Y_{\lambda_{n}}, X}\left\|y_{n}\right\|_{Y_{\lambda_{n}}} \\
& \leq \frac{\delta}{2}+\left(\sum_{n=N+1}^{\infty}\left(\|T\|_{Y_{\lambda_{n}}, X} / \psi\left(2^{-n}\right)\right)^{q^{\prime}}\right)^{1 / q^{\prime}}\left(\sum_{n=N+1}^{\infty}\left(\psi\left(2^{-n}\right)\left\|y_{n}\right\|_{Y_{\lambda_{n}}}\right)^{q}\right)^{1 / q} \\
& \leq \frac{\delta}{2}+\frac{\delta}{4} \cdot 2=\delta
\end{aligned}
$$

Consequently,

$$
T\left(U_{Y_{\theta, \psi, q}^{-}}\right) \subseteq \delta U_{X}+R\left(U_{E}\right)
$$

which completes the proof.

Writing down Theorems 3.1 and 3.2 for $\mathcal{A}=\mathcal{K}$, the ideal of compact operators, we recover results of Cobos and Kühn [8, Thms. 4.1 and 4.2]. Our theorems can be also applied to weakly compact operators, Banach-Saks operators, Rosenthal operators or Asplund operators. We can also apply Theorem 3.1 to strictly singular operators and Theorem 3.2 to strictly cosingular operators.

\section{Extrapolation on the source spaces and on the domain spaces}

For the results of this section we shall require that the ideal $\mathcal{A}$ satisfies the socalled $\Sigma_{q}$-condition that we recall next. Given any sequence of Banach spaces $\left(E_{n}\right)$ and any sequence of positive numbers $\left(\mu_{n}\right)$, we denote by $\ell_{q}\left(\mu_{n} E_{n}\right)$ the vector valued $\ell_{q}$-space formed by all sequences $x=\left(x_{n}\right)$ with $x_{n} \in E_{n}$ and

$$
\|x\|_{\ell_{q}\left(\mu_{n} E_{n}\right)}=\left(\sum_{n}\left(\mu_{n}\left\|x_{n}\right\|_{E_{n}}\right)^{q}\right)^{1 / q}<\infty .
$$

We write $Q_{k}: \ell_{q}\left(\mu_{n} E_{n}\right) \rightarrow \mu_{k} E_{k}$ for the projection $Q_{k}\left(x_{n}\right)=x_{k}$, and $P_{k}: \mu_{k} E_{k} \rightarrow$ $\ell_{q}\left(\mu_{n} E_{n}\right)$ for the embedding $P_{k} x=\left(\delta_{n}^{k} x\right)$ where $\delta_{n}^{k}$ is the Kronecker delta.

Definition 4.1. Let $1<q<\infty$. An operator ideal $\mathcal{A}$ is said to satisfy the $\Sigma_{q}$-condition if for any sequences of Banach spaces $\left(E_{n}\right),\left(F_{n}\right)$ and any operator $T \in \mathcal{L}\left(\ell_{q}\left(E_{n}\right), \ell_{q}\left(F_{n}\right)\right)$, it follows from $Q_{k} T P_{n} \in \mathcal{A}\left(E_{n}, F_{k}\right)$ for any $n, k$ that $T \in \mathcal{A}\left(\ell_{q}\left(E_{n}\right), \ell_{q}\left(F_{n}\right)\right)$.

As it has been pointed out by Heinrich [16], every ideal which satisfies the $\Sigma_{q^{-}}$ condition is closed. It is also proved in [16] that weakly compact operators, BanachSaks operators, Rosenthal operators and Asplund operators satisfy the $\Sigma_{q}$-condition for any $1<q<\infty$. On the other hand, it is easy to see that compact operators does not satisfy the $\Sigma_{q}$-condition for any $q$. We refer to [4] and [14] for more information on this condition and its extension to Banach lattices of sequences.

Let $X_{0} \hookrightarrow X_{\eta} \hookrightarrow X_{1}$ and $Y_{0} \hookrightarrow Y_{\eta} \hookrightarrow Y_{1}$ be ordered families with $\eta \in \Theta \subseteq[0,1]$. We suppose that in each family embedding operators are uniformly bounded. Let $1<q<\infty$ and assume that $\theta, \varepsilon$ and $\varphi$ are as in Definition 2.1 or $\theta, \varepsilon$ and $\psi$ are as in Definition 2.2. By $T \in \mathcal{L}\left(\left\{X_{\eta}\right\}_{\eta \in \Theta},\left\{Y_{\eta}\right\}_{\eta \in \Theta}\right)$ we mean that $T$ is a linear operator whose restrictions

$$
T: X_{1} \rightarrow Y_{1}, \quad T: X_{0} \rightarrow Y_{0} \quad \text { and } \quad T: X_{\eta} \rightarrow Y_{\eta}
$$


are bounded with $\sup _{\eta \in \Theta}\|T\|_{X_{\eta}, Y_{\eta}}<\infty$. Then it is easy to check that restrictions

$$
T: X_{\theta, \varphi, q}^{+} \rightarrow Y_{\theta, \varphi, q}^{+} \quad \text { and } \quad T: X_{\theta, \psi, q}^{-} \rightarrow Y_{\theta, \psi, q}^{-}
$$

are also bounded. Next we show sufficient conditions for restrictions (4.1) to belong to an operator ideal $\mathcal{A}$ with the $\Sigma_{q}$-property.

Theorem 4.2. Let $1<q<\infty$ and let $\mathcal{A}$ be an injective operator ideal which satisfies the $\Sigma_{q}$-condition. Suppose that $\left\{X_{\eta}\right\}_{\eta \in \Theta},\left\{Y_{\eta}\right\}_{\eta \in \Theta}$ are ordered families with uniformly bounded embeddings and let $\theta, \varepsilon$ and $\varphi$ be as in Definition 2.1. If $T \in$ $\mathcal{L}\left(\left\{X_{\eta}\right\}_{\eta \in \Theta},\left\{Y_{\eta}\right\}_{\eta \in \Theta}\right)$ and

$$
T \in \mathcal{A}\left(X_{\eta}, Y_{\tau}\right) \text { for any } \eta<\tau \text { with } \eta, \tau \in(\theta, \theta+\varepsilon)
$$

then

$$
T: X_{\theta, \varphi, q}^{+} \rightarrow Y_{\theta, \varphi, q}^{+} \text {belongs to } \mathcal{A} \text {. }
$$

Proof. Take any $\theta<\eta<\theta+\varepsilon$. By (2.5) and (4.2), for any $\tau \in(\eta, \theta+\varepsilon)$, we have that

$$
T: X_{\theta, \varphi, q}^{+} \rightarrow Y_{\tau} \text { belongs to } \mathcal{A} \text {. }
$$

Choose $J \in \mathbf{N}$ with $2^{-J}<\varepsilon$ and let $\sigma_{k}$ be the numbers defined by (2.4). We consider on $Y_{\theta, \varphi, q}^{+}$the discrete norm $\|\cdot\|_{Y_{\theta, \varphi, q}^{+}}$. The map $j: Y_{\theta, \varphi, q}^{+} \rightarrow \ell_{q}\left(\varphi\left(2^{-n}\right) Y_{\sigma_{n}}\right)$ defined by $j y=(y, y, \ldots)$ is an isometric embedding. For any $k \geq N$, it follows from (4.3) that

$$
Q_{k} j T \in \mathcal{A}\left(X_{\theta, \varphi, q}^{+}, \varphi\left(2^{-k}\right) Y_{\sigma_{k}}\right) .
$$

Since $\mathcal{A}$ satisfies the $\Sigma_{q}$-condition, we obtain that $j T \in \mathcal{A}\left(X_{\theta, \varphi, q}^{+}, \ell_{q}\left(\varphi\left(2^{-n}\right) Y_{\sigma_{n}}\right)\right)$. Finally, using that $\mathcal{A}$ is injective, we conclude that $T \in \mathcal{A}\left(X_{\theta, \varphi, q}^{+}, Y_{\theta, \varphi, q}^{+}\right)$.

Theorem 4.3. Let $1<q<\infty$ and let $\mathcal{A}$ be a surjective operator ideal which satisfies the $\Sigma_{q}$-condition. Suppose that $\left\{X_{\eta}\right\}_{\eta \in \Theta},\left\{Y_{\eta}\right\}_{\eta \in \Theta}$ are ordered families with uniformly bounded embeddings and let $\theta, \varepsilon$ and $\psi$ be as in Definition 2.2. If $T \in$ $\mathcal{L}\left(\left\{X_{\eta}\right\}_{\eta \in \Theta},\left\{Y_{\eta}\right\}_{\eta \in \Theta}\right)$ and

$$
T \in \mathcal{A}\left(X_{\eta}, Y_{\tau}\right) \text { for any } \eta<\tau \text { with } \eta, \tau \in(\theta-\varepsilon, \theta),
$$

then

$$
T: X_{\theta, \psi, q}^{-} \rightarrow Y_{\theta, \psi, q}^{-} \text {belongs to } \mathcal{A} \text {. }
$$

Proof. Using (2.10) and (4.4), we get that

$$
T: X_{\eta} \rightarrow Y_{\theta, \psi, q}^{-} \text {belongs to } \mathcal{A} \text { for any } \eta \in(\theta-\varepsilon, \theta) \text {. }
$$

Let again $J \in \mathbf{N}$ with $2^{-J}<\varepsilon$, let $\lambda_{n}$ be the numbers defined by (2.8) and consider the metric surjection $\pi: \ell_{q}\left(\psi\left(2^{-n}\right) X_{\lambda_{n}}\right) \rightarrow Y_{\theta, \psi, q}^{-}$, defined by $\pi\left(x_{n}\right)=\sum_{n \geq J} x_{n}$ (convergence in $\left.X_{1}\right)$. For any $k \geq N$,(4.5) implies that $T \pi Q_{k}: \psi\left(2^{-k}\right) X_{\lambda_{k}} \rightarrow Y_{\theta, \psi, q}^{-}$belongs to $\mathcal{A}$. Since $\mathcal{A}$ satisfies the $\Sigma_{q}$-condition, it follows that $T \pi \in \mathcal{A}\left(\ell_{q}\left(\psi\left(2^{-n}\right) X_{\lambda_{n}}\right), Y_{\theta, \psi, q}^{-}\right)$. Then, surjectivity of $\mathcal{A}$ yields that $T \in \mathcal{A}\left(X_{\theta, \psi, q}^{-}, Y_{\theta, \psi, q}^{-}\right)$.

Often scales are constructed by interpolation. Next we show that if this is the case, then we can use the known results on interpolation of closed operator ideals (see [16], [7] and [4]) with the effect that assumptions on $T$ in the previous results can be weakened. Let $X_{0}, X_{1}, Y_{0}, Y_{1}$ be Banach spaces with $X_{0} \hookrightarrow X_{1}, Y_{0} \hookrightarrow Y_{1}$ and embeddings having norm less than or equal to 1 . For $0 \leq \eta \leq 1$, let $X_{\eta}=\left[X_{0}, X_{1}\right]_{\eta}$, $Y_{\eta}=\left[Y_{0}, Y_{1}\right]_{\eta}$ be the complex interpolation spaces generated by the given couples 
(see [3], [28]). Then $\left\{X_{\eta}\right\}_{0 \leq \eta \leq 1},\left\{Y_{\eta}\right\}_{0 \leq \eta \leq 1}$ are ordered families, with embedding operators having norms $\leq 1$. Given any linear operator $T \in \mathcal{L}\left(X_{1}, Y_{1}\right) \cap \mathcal{L}\left(X_{0}, Y_{0}\right)$, the interpolation property of the complex method yields that $T \in \mathcal{L}\left(X_{\eta}, Y_{\eta}\right)$ for any $0<\eta<1$, with $\sup _{0<\eta<1}\|T\|_{X_{\eta}, Y_{\eta}}<\infty$. We also recall the reiteration formula for the complex method (see [3] and [10]): If $0 \leq \eta_{0}, \eta_{1}, \mu \leq 1$ and $\eta=(1-\mu) \eta_{0}+\mu \eta_{1}$ then we have with equality of norms

$$
\left[\left[X_{0}, X_{1}\right]_{\eta_{0}},\left[X_{0}, X_{1}\right]_{\eta_{1}}\right]_{\mu}=\left[X_{0}, X_{1}\right]_{\eta} .
$$

Corollary 4.4. Let $1<q<\infty$ and let $\mathcal{A}$ be an injective and surjective operator ideal which satisfies the $\Sigma_{q}$-condition. Let $0 \leq \theta<1,0<\varepsilon<1$ and let $\varphi$ be a positive, continuous and monotone function on $(0, \varepsilon)$ with $\varphi(t) \sim \varphi(2 t)$ and $\left(\int_{0}^{\varepsilon} \varphi(t)^{q} d t / t\right)^{1 / q}<\infty$. Suppose that $X_{0}, X_{1}, Y_{0}, Y_{1}$ are Banach spaces such that $X_{0} \hookrightarrow X_{1}, Y_{0} \hookrightarrow Y_{1}$ with embeddings having norm $\leq 1$. Let $X_{\eta}=\left[X_{0}, X_{1}\right]_{\eta}$ and $Y_{\eta}=\left[Y_{0}, Y_{1}\right]_{\eta}$. If

$$
T \in \mathcal{L}\left(X_{1}, Y_{1}\right) \cap \mathcal{L}\left(X_{0}, Y_{0}\right) \quad \text { and } \quad T \in \mathcal{A}\left(X_{0}, Y_{1}\right)
$$

then

$$
T: X_{\theta, \varphi, q}^{+} \rightarrow Y_{\theta, \varphi, q}^{+} \text {belongs to } \mathcal{A} \text {. }
$$

Proof. Take any $\eta$ and $\tau$ with $\theta<\eta<\tau<\theta+\varepsilon$. As it is pointed out in [3] and [28], the complex interpolation space $X_{\eta}$ is of class $\mathcal{C}(\eta ; \bar{X})$. Having this is mind, it follows from the fact that $T \in \mathcal{L}\left(X_{1}, Y_{1}\right) \cap \mathcal{A}\left(X_{0}, Y_{1}\right)$, sobrejectivity of $\mathcal{A}$ and [16, Prop. 1.7] that $T \in \mathcal{A}\left(X_{\eta}, Y_{1}\right)$. Now, consider the diagram

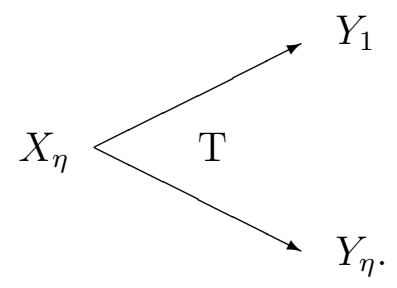

By injectivity of $\mathcal{A}$, the reiteration formula and [16, Prop. 1.6], we derive that $T \in$ $\mathcal{A}\left(X_{\eta}, Y_{\tau}\right)$. Consequently, the result follows from Theorem 4.2.

Similarly, but using now Theorem 4.3, we obtain the following.

Corollary 4.5. Let $1<q<\infty$ and let $\mathcal{A}$ be an injective and surjective operator ideal which satisfies the $\Sigma_{q}$-condition. Let $0<\theta \leq 1,0<\varepsilon<1$ and let $\psi$ be a positive, continuous and monotone function on $(0, \bar{\varepsilon})$ with $\psi(t) \sim \psi(2 t)$ and $\left(\int_{0}^{\varepsilon} \psi(t)^{-q^{\prime}} d t / t\right)^{1 / q^{\prime}}<\infty, 1 / q+1 / q^{\prime}=1$. Suppose that $X_{0}, X_{1}, Y_{0}, Y_{1}$ are Banach spaces such that $X_{0} \hookrightarrow X_{1}, Y_{0} \hookrightarrow Y_{1}$ with embeddings having norm $\leq 1$. Let $X_{\eta}=\left[X_{0}, X_{1}\right]_{\eta}$ and $Y_{\eta}=\left[Y_{0}, Y_{1}\right]_{\eta}$. If

$$
T \in \mathcal{L}\left(X_{1}, Y_{1}\right) \cap \mathcal{L}\left(X_{0}, Y_{0}\right) \text { and } T \in \mathcal{A}\left(X_{0}, Y_{1}\right)
$$

then

$$
T: X_{\theta, \psi, q}^{-} \rightarrow Y_{\theta, \psi, q}^{-} \text {belongs to } \mathcal{A} \text {. }
$$

The special case $\varphi(t)=t^{b}$ and $\psi(t)=t^{-b}$, with $b>0$, corresponds to the socalled logarithmic spaces in [12] and [13]. As an example, let us recall that if $\Omega$ is a bounded open subset of $\mathbf{R}^{d}$, and we put $X_{0}=L_{\infty}(\Omega), X_{1}=L_{1}(\Omega)$, then we have that $\left[L_{\infty}(\Omega), L_{1}(\Omega)\right]_{\theta}=L_{1 / \theta}(\Omega)$, and for $1<p<\infty$ we obtain

$$
X_{1 / p, b, p}^{+}=L_{p}(\log L)_{-b}(\Omega) \quad \text { and } \quad X_{1 / p, b, p}^{-}=L_{p}(\log L)_{b}(\Omega)
$$


(see [12, Section 2.6.2]). Here, for $\gamma \in \mathbf{R}, L_{p}(\log L)_{\gamma}(\Omega)$ is the usual Zygmund space (see [2]).

For logarithmic spaces the following holds.

Corollary 4.6. Let $1<q<\infty$ and let $\mathcal{A}$ be an injective and surjective operator ideal which satisfies the $\Sigma_{q}$-condition. Let $b>0$ and assume that $X_{0}, X_{1}, Y_{0}, Y_{1}$ are Banach spaces such that $X_{0} \hookrightarrow X_{1}, Y_{0} \hookrightarrow Y_{1}$ with embeddings having norm $\leq 1$. Let $X_{\eta}=\left[X_{0}, X_{1}\right]_{\eta}, Y_{\eta}=\left[Y_{0}, Y_{1}\right]_{\eta}$ and let $T \in \mathcal{L}\left(X_{1}, Y_{1}\right) \cap \mathcal{L}\left(X_{0}, Y_{0}\right)$.

(a) For $0 \leq \theta<1$, the necessary and sufficient condition for $T: X_{\theta, b, q}^{+} \rightarrow Y_{\theta, b, q}^{+}$to belong to $\mathcal{A}$ is that $T \in \mathcal{A}\left(X_{0}, Y_{1}\right)$.

(b) For $0<\theta \leq 1$, the necessary and sufficient condition for $T: X_{\theta, b, q}^{-} \rightarrow Y_{\theta, b, q}^{-}$to belong to $\mathcal{A}$ is that $T \in \mathcal{A}\left(X_{0}, Y_{1}\right)$.

Proof. Sufficiently follows from Corollaries 4.4 and 4.5. Necessity is a consequence of the factorizations

$$
X_{0} \hookrightarrow X_{\theta, b, q}^{+} \stackrel{T}{\rightarrow} Y_{\theta, b, q}^{+} \hookrightarrow Y_{1}, \quad X_{0} \hookrightarrow X_{\theta, b, q}^{-} \stackrel{T}{\rightarrow} Y_{\theta, b, q}^{-} \hookrightarrow Y_{1} .
$$

Writing down Corollary 4.6 for the case of weakly compact operators, we obtain a result which improves [24, Cor. 2.3]: It is enough that $T: X_{0} \rightarrow Y_{1}$ belongs to $\mathcal{W}$, instead of requiring that $T \in \mathcal{W}\left(X_{j}, Y_{j}\right)$ for $j=0$ or $j=1$.

For the particular case when $T$ is the identity operator we conclude the following result.

Corollary 4.7. Let $1<q<\infty$ and let $\mathcal{A}$ be an injective and surjective operator ideal which satisfies the $\Sigma_{q}$-condition. Let $b>0$, assume that $X_{0}, X_{1}$ are Banach spaces such that the embedding $X_{0} \hookrightarrow X_{1}$ belongs to $\mathcal{A}$, and let $X_{\eta}=\left[X_{0}, X_{1}\right]_{\eta}$.

(a) For $0 \leq \theta<1$, the identity operator of $X_{\theta, b, q}^{+}$belongs to $\mathcal{A}$.

(b) For $0<\theta \leq 1$, the identity operator of $X_{\theta, b, q}^{-,}$also belongs to $\mathcal{A}$.

As a direct consequence of Corollary 4.7, if the embedding $X_{0} \hookrightarrow X_{1}$ is weakly compact, we derive that for $0<\theta<1,1<q<\infty$ and $b>0$, spaces $X_{\theta, b, q}^{+}$and $X_{\theta, b, q}^{-}$ are reflexive. This result improves [24, Cor. 2.2/(ii)]: No assumption of density of $X_{0}$ in $X_{1}$ is required and it is enough that the embedding $X_{0} \hookrightarrow X_{1}$ belongs to $\mathcal{W}$, instead of requiring the stronger assumption that $X_{0}$ or $X_{1}$ is reflexive.

We refer to $[22,23,24]$ for other geometrical properties of logarithmic spaces.

\section{References}

[1] Astala, K.: On measures of non-compactness and ideal variations in Banach spaces. - Ann. Acad. Sci. Fenn. Ser. A I Math. Diss. 29, 1980, 1-42.

[2] Bennett, C., and R. Sharpley: Interpolation of operators. - Academic Press, Boston, 1988.

[3] Bergh, J., and J. LÖfström: Interpolation spaces. An introduction. - Springer, Berlin, 1976.

[4] Cobos, F., L. M. Fernández-Cabrera, A. Manzano, and A. Martínez: Real interpolation and closed operator ideals. - J. Math. Pures Appl. 83, 2004, 417-432.

[5] Cobos, F., L. M. Fernández-Cabrera, A. Manzano, and A. Martínez: Logarithmic interpolation spaces between quasi-Banach spaces. - Z. Anal. Anwend. 26, 2007, 65-86.

[6] Cobos, F., L. M. Fernández-Cabrera, and H. Triebel: Abstract and concrete logarithmic interpolation spaces. - J. London Math. Soc. 70, 2004, 231-243.

[7] Cobos, F., A. Manzano, and A. Martínez: Interpolation theory and measures related to operator ideals. - Quart. J. Math. 50, 1999, 401-416. 
[8] CoBos, F., and T. KÜHn: Extrapolation results of Lions-Peetre type. - Preprint, 2012.

[9] Cobos, F., and T. Kühn: Extrapolation estimates for entropy numbers. - J. Funct. Anal. 263, 2012, 4009-4033.

[10] Cwikel, M.: Complex interpolation spaces, a discrete definition and reiteration. - Indiana Univ. Math. J. 27, 1978, 1005-1009.

[11] Diestel, J., H. Jarchow, and A. Tonge: Absolutely summing operators. - Cambridge Univ. Press, Cambridge, 1995.

[12] Edmunds, D. E., and H. Triebel: Function spaces, entropy numbers, differential operators. - Cambridge Univ. Press, Cambridge, 1996.

[13] Edmunds, D. E., and H. Triebel: Logarithmic spaces and related trace problems. - Functiones et Approximatio 26, 1998, 189-204.

[14] Fernández-Cabrera, L. M., and A. Martínez: Interpolation of ideal measures by abstract K and J spaces. - Acta Math. Sin. (Engl. Ser.) 23, 2007, 1357-1374.

[15] Fiorenza, A., and G. E. Karadzhov: Grand and small Lebesgue spaces and their analogs. - Z. Anal. Anwend. 23, 2004, 657-681.

[16] Heinrich, S.: Closed operator ideals and interpolation. - J. Funct. Anal. 35, 1980, 397-411.

[17] Jawerth, B., and M. Milman: Extrapolation theory with applications. - Mem. Amer. Math. Soc. 89:440, 1991.

[18] Jarchow, H.: Locally conves spaces. - Teubner, Stuttgart, 1981.

[19] KÜhn, T., and T. Schonbek: Extrapolation of entropy numbers. - Contemp. Math. 445, 2007, 195-206.

[20] KRYcZKA, A.: Weak noncompactness in Banach sequence spaces and its extrapolation properties. - Math. Inequl. Appl. 11, 2008, 297-306.

[21] Milman, M.: Extrapolation and optimal decompositions. - Lecture Notes in Math. 1580, Springer, Berlin, 1994.

[22] Nikolova, L., L. E. Persson, and T. Zachariades: On Clarkson's inequalities, type and cotype for the Edmunds-Triebel logarithmic spaces. - Arch. Math. (Basel) 80, 2003, 165-176.

[23] Nikolova, L., and T. Zachariades: The uniform convexity of the Edmunds-Triebel logarithmic spaces. - J. Math. Anal. Appl. 283, 2003, 549-556.

[24] Nikolova, L., and T. Zachariades: On Edmunds-Triebel spaces. - Banach J. Math. Anal. 4, 2010, 146-158.

[25] Pietsch, A.: Operator ideals. - North-Holland, Amsterdam, 1980.

[26] Stein, E. M.: Singular integrals and differentiability properties of functions. - Princeton Univ. Press, Princeton, 1970.

[27] Szwedek, R.: Interpolation of a measure of weak non-compactness. - Ann. Acad. Sci. Fenn. Math. 36, 2011, 537-552.

[28] Triebel, H.: Interpolation theory, function spaces, differential operators. - North-Holland, Amsterdam, 1978.

[29] Triebel, H.: Theory of function spaces II. - Birkhäuser, Basel, 1992.

[30] Triebel, H.: Approximation numbers and entropy numbers of embeddings of fractional Besov-Sobolev spaces in Orlicz spaces. - Proc. London Math. Soc. 66, 1993, 589-618.

[31] Tylli, H.-O.: The essential norm of an operator is not self-dual. - Israel J. Math. 91, 1995, 93-110. 